\title{
Diacronie
}

Studi di Storia Contemporanea

$N^{\circ} 14,2 \mid 2013$

Processo penale, politica, opinione pubblica (secoli XVIII-XX)

\section{Raniero Paulucci di Calboli e l'affaire Dreyfus}

\section{Francesco Gioiello}

\section{(2) OpenEdition \\ Journals}

Edizione digitale

URL: http://journals.openedition.org/diacronie/131

DOI: 10.4000/diacronie.131

ISSN: 2038-0925

Editore

Association culturelle Diacronie

Notizia bibliografica digitale

Francesco Gioiello, «Raniero Paulucci di Calboli e l'affaire Dreyfus », Diacronie [Online], № 14, 2 | 2013, documento 2, Messo online il 01 août 2013, consultato il 30 avril 2019. URL : http://

journals.openedition.org/diacronie/131; DOI : 10.4000/diacronie.131 


\section{Diacronie}

\section{2/}

\section{Raniero Paulucci di Calboli e l'affaire Dreyfus}

Francesco GIOIELLO*

L'affaire Dreyfus rappresenta emblematicamente la dimensione ancipite del diritto: prima arma per colpire il nemico comune Dreyfus, poi strumento di difesa brandito dagli intellettuali engages - con Zola in prima fila - per chiedere giustizia e far trionfare la verità. Si rese conto di tutto ciò Raniero Paulucci di Calboli, diplomatico forlivese che operò nella Parigi dell'affaire Dreyfus.

Attraverso il suo diario del 1898 ed il fondo costituito dallo stesso a partire dal 1897 (oggi conservato presso la biblioteca "A. Saffi" di Forlì) vengono qui ricostruite le vicende processuali, ma si evidenzia anche il ruolo giocato dagli avvocati, dagli intellettuali e dalla stampa, nonché il peso dell'opinione pubblica, in questa complessa vicenda, che ancora oggi non cessa di far discutere.

\section{Introduzione}

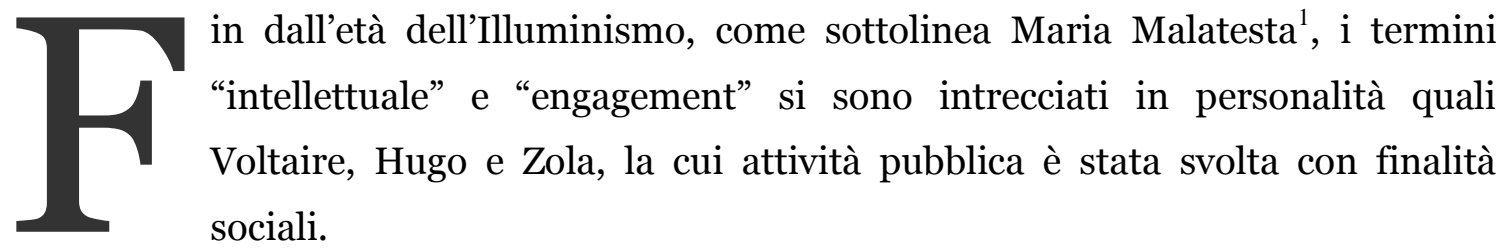

Scrive in proposito Malatesta: «Questi scrittori rappresentarono un modello di intellettuale la cui universalità era data dal fatto di possedere una verità ritenuta estranea al potere e di sapere tradurre - grazie alla loro cultura - le istanze provenienti dal basso».

Dopo il 1968 la nozione di intellettuale cambiò radicalmente: dall'“intellettuale universale" si passò infatti all'“intellettuale specifico" (concettualmente definito da

\footnotetext{
${ }^{1}$ Si veda la presentazione di Maria Malatesta in ISRAËL, Liora, Le armi del diritto, Milano,
} Giuffrè, 2012, p. VII. 
Foucault nel 1971), rappresentato dall'esperto operante nei differenti ambiti (sanitario, giuridico, tecnico) ossia il medico, l'architetto, l'avvocato e il magistrato. Proprio queste ultime due categorie in particolare, possono sfruttare in maniera ottimale quelle che vengono definite, utilizzando il titolo di un saggio di Liora Israël, "le armi del diritto", «in difesa dei fondamenti sociali, politici e antropologici della democrazia»².

Il diritto, avverte Israël, è «sia arma offensiva, per far valere dei diritti, sia arma di difesa, perché imposta da una indagine o da una imputazione»3, per contestare una situazione. In questo ambito certamente già rientrava, in modo emblematico, l'affaire Dreyfus.

In tale vicenda, infatti, la dimensione del diritto è ben chiara: prima si tentò di brandire l'arma del diritto (da parte di esercito e governo) per colpire e reprimere il nemico comune Dreyfus, nel nome del nazionalismo e dell'antisemitismo e a difesa della cosiddetta Terza Repubblica, borghese e conservatrice. Poi, però, lo stesso diritto venne utilizzato come arma di difesa dagli intellettuali engagés (con Zola in prima fila) e - sia pure con un ruolo secondario - dagli avvocati quale strumento per chiedere giustizia e far trionfare la verità4.

Questi in estrema sintesi sono i fatti del celebre affaire: nel 1894 Alfred Dreyfus venne arrestato con l'accusa di alto tradimento per aver passato alla Germania il famoso bordereau. Processato e condannato, venne degradato ed esiliato perpetuamente sull'isola del Diavolo. Nel marzo del 1896 il tenente colonnello Picquart iniziò a dubitare della colpevolezza di Dreyfus ed anche gli intellettuali si mobilitarono: il vero colpevole fu individuato nel maggiore Esterhazy. Questi fu sottoposto a processo, ma venne però assolto; a questo punto Zola pronunciò il suo J'accuse, il 13 gennaio 1898, su «L'Aurore». Dreyfus ottenne la revisione del processo nel settembre 1899, ma fu riconosciuto colpevole una seconda volta; dieci giorni dopo, tuttavia, il Presidente della Repubblica gli concesse la grazia. Solo nel 1906, infine, la sentenza venne annullata e Dreyfus ottenne il reintegro nell'esercito.

Il caso Dreyfus non fu certo stato l'unico, dall'avvento dell'Illuminismo, in cui si registrò un uso politico dell'avvocatura: basterà qui ricordare il "caso Calas" 5 . Con esso, per la prima volta, un processo penale divenne un affaire: colui che era imputato

\footnotetext{
2 Ibidem.

3 Ibidem, p. 3.

4 «L'atto che compio oggi non è che un mezzo rivoluzionario per sollecitare l'esplosione della verità e della giustizia». ZOLA, Émile, L'affaire Dreyfus - La verità in cammino, Firenze, Casa Editrice Giuntina, 2011, p. VII.

5 PONTIERI, Enrico, «Opinion publique e nozione di affaire nella Francia del XVIII secolo: Voltaire difensore di Calas ", Diacronie. Studi di Storia Contemporanea: La parola alla difesa, URL: <http://www.studistorici.com/2013/o8/29/pontieri_numero_14/> [consultato il 29 agosto 2013].
} 
divenne vittima di un sistema e un intellettuale universale, in questo caso Voltaire, lo trasformò da denunciato in garante, per stigmatizzare un sistema non più sopportabile.

Ma una simile concezione dell'importanza del processo, divenuto politico, era già chiara in Raniero Paulucci di Calboli, giovane diplomatico forlivese che operò nella Parigi dell'affaire Dreyfus, il quale nel 1930 avrebbe scritto:

Quando si esamini la storia moderna di Francia ci si imbatte sovente in una serie di processi che tendono a trasformare sensibilmente la psicologia politica di tutto il paese. Vediamo così l'affare Calas, quello del collare della Regina, e infine l'affare Dreyfus che ne sono la prova più evidente. [...] In Francia dei tre affari sopra ricordati quello Calas fu certo il solo motivo che dette nel 1765 al Voltaire oltre alla riabilitazione della povera vittima, il diritto di poter enunciare i sommi principii di libertà della grande rivoluzione. Il processo posteriore del collare contro il cardinale principe di Rohan e la contessa Lamotte, li rese di più facile applicazione. L'affare Dreyfus, infine, ha convertito la vecchia repubblica moderata francese in un Governo radicale ${ }^{6}$.

Rilevò come si fosse passati dalla Repubblica degli avvocati a quella degli intellettuali. Tale consapevolezza, sia pure ad un livello embrionale, era già riscontrabile nelle pagine del dettagliato diario che Paulucci redasse nel 18987; infatti, in data 19 gennaio, appare chiaro come l'affaire da «questione giuridica sia cambiata adesso in politica e sociale» 8 . E ancora ( 23 febbraio): «Che disonore per un paese quello di avere un potere militare così dispotico e brutale, e una magistratura civile così bassamente subordinata ai voleri del più forte»9.

Fine conoscitore delle cose di Francia, Paulucci diede prova del suo acume anche in un'interessante nota inviata al Ministero con la denominazione Situazione interna della Francia, datata 18 novembre $1905^{10}$; in essa, analizzando i candidati alla Presidenza della Repubblica in prossimità delle elezioni, si legge a proposito di Fallières (che sarebbe poi risultato vincitore): «Vecchio innanzi tempo, pieno di acciacchi, privo

6 PAULUCCI DI CALBOLI, Raniero, Le mie impressioni sull'affare Dreyfus, in Raniero Paulucci di Calboli dans le Paris de l'affaire Dreyfus, (a cura di TASSANI, Giovanni), Forlì, Guaraldi, 1995, p. 141.

7 PAULUCCI DI CALBOLI, Raniero (a cura di TASSANI, Giovanni), Parigi 1898 con Zola, per Dreyfus. Diario di un diplomatico, Bologna, CLUEB, 1998.

8 Ibidem, p. 46.

9 Ibidem, p. 72.

${ }^{10}$ PAULUCCI DI CALBOLI, Raniero, Situazione interna della Francia, Elezione del presidente della Repubblica, Parigi, 21 novembre 1905, documento dattilografato di 21 pp., inviato con lettera di 4 pp. al ministro degli Esteri Tittoni dall'ambasciatore Tornielli, in Archivio Paulucci di Calboli, Archivio di Stato, Forlì. 
di energia fisica e morale [...] i servizi resi da questo vecchio repubblicano, se non la sua mediocrità, sola potenza che abbia la virtù di neutralizzare l'invidia, additano il nome del Fallières alla maggioranza del Senato. [...] le probabilità di riuscita di questo pretendente appaiono quindi moltissime». L'affaire viene ripetutamente citato, a sottolineare quanto abbia segnato in profondità la storia politica francese; a proposito di Doumer (altro pretendente alla Presidenza) si dice infatti che la fortuna lo allontanò «di Francia nel doloroso periodo di quell'affaire, che compromise la sorte di tanti uomini politici, conservandogli [...] verginità» ${ }^{11}$. Di contro, si indica in Charles Dupuy «una delle tante personalità rovinate dall'affaire»12 per poi concludere «tutto lascia credere infine che la prossima riunione plenaria di Versaglia, a differenza di quella del febbraio 1899, sarà calma e tranquilla e [...] consoliderà sempre viepiù quella tranquillità di cui la Francia sente dopo tante convulsioni, il bisogno»13. Ancora una volta, il riferimento al caso Dreyfus - sia pure soggiaciuto - appare scontato.

Paulucci fa inoltre capire quanto fosse a conoscenza della politica francese nel documento da lui stilato riguardante L'Alleanza Franco-Russa, anch'esso del 1905 ${ }^{14}$. Il diplomatico spiegò come e perché la Francia, bisognosa di revanche, avesse scelto come partner privilegiato proprio la Russia, col supporto fondamentale della stampa, per influenzare l'opinione pubblica. Quest'ultima era infatti riuscita ad avere ancora una volta la meglio, esattamente come nel caso Dreyfus; così, malgrado l'opposizione di repubblicani e liberali, nel 1897 l'alleanza era stata firmata. Un'ulteriore annotazione riguarda il ruolo del partito militare, "potentissimo non tanto per le aderenze di classe, quanto per quell'influsso d'innato spirito guerresco che è il fondo del carattere dei nove decimi della popolazione» ${ }^{15}$, che tanto influenzò il caso Dreyfus; contro di esso, il partito degli intellettuali non poteva opporre la compattezza necessaria («come lo ha dimostrato nella lunga ed ardita campagna del celebre Affaire»16) per guidare l'opinione pubblica.

Appare perciò particolarmente interessante ripercorrere i momenti salienti dell'affaire Dreyfus attraverso le riflessioni contenute nel diario già ricordato, ma anche

${ }^{11}$ Ibidem, p. 8.

12 Ibidem, p. 19.

13 Ibidem.

14 PAULUCCI DI CALBOLI, Raniero, L'alleanza Franco-Russa, Parigi, s.e., 1905, in Archivio Ministero Affari Esteri, Roma (ottenuto in fotocopia da Giovanni Tassani).

15 Ibidem, p. 5 .

${ }_{16}$ Ibidem, p. 7. 
attraverso la documentazione presente nel fondo (conservato presso la biblioteca comunale "A. Saffi” di Forlì) che lo stesso Paulucci ha nel tempo collezionato ${ }^{17}$.

\section{L'affaire Dreyfus: i processi del 1894 e del 1899 e la riabilitazione del 1906}

La conclusione della complessa vicenda giudiziaria (non documentata da Paulucci, nel frattempo trasferito a Lisbona) sarebbe avvenuta il 12 luglio 1906, con l'annullamento da parte della Corte di Cassazione della sentenza di Rennes contro Dreyfus. In precedenza si era invece svolto il processo di Rennes, protrattosi dal 7 agosto al 9 settembre 1899: si trattava della revisione della sentenza del processo del 1894; di quest'ultimo non vi è traccia nel fondo, ma ugualmente ne riportiamo i tratti più significativi.

Il Ministro della Guerra Mercier, convinto del tradimento di Dreyfus, perseverava costantemente nel suo errore; l'Ufficio di Statistica (il controspionaggio dell'epoca) e alcuni ufficiali realizzarono su suo ordine il noto dossier segreto (che venne accresciuto dopo il 1894 fino a comprendere circa 500 documenti), poi presentato alla Corte di Cassazione alla fine del 1898; in esso, numerosi sono i falsi. Recentemente i tre ricercatori Gervais, Peretz e Stutin ${ }^{18}$ hanno tentato di ricostruire l'accusa che rese possibile condannare un innocente senza prove di colpevolezza. Le colpe e le responsabilità dei militari e della classe politica, influenzati dal nazionalismo, dalla xenofobia e dall'antisemitismo, paiono evidenti. Non si trattò, quindi, solo di un errore giudiziario, ma di un crimine di Stato e il processo assunse perciò valenza politica.

Nel caso Dreyfus però troviamo una doppia novità: il giudizio fu emesso da un tribunale militare (il Consiglio di Guerra), non da un tribunale civile, e gli atti segreti vennero comunicati ai giudici durante la delibera finale e non nel corso dell'istruttoria preliminare. L'esito del processo costituì una sorpresa: all'unanimità Dreyfus viene riconosciuto colpevole di alto tradimento.

L'opinione pubblica e la stampa contribuirono a creare contro il capitano un'ostilità ferocea; egli fu mandato sull'isola Saint Martin de Ré (il 17 gennaio), prima di essere inviato (il 9 febbraio) sulle Îles du Salut (Guyane) e infine trasferito sull'̂̂le du Diable.

17 MILZA, Pierre (a cura di), Dreyfus. L'affaire e la Parigi fin de siècle nelle carte di un diplomatico italiano, Roma, Edizioni Lavoro, 1994.

18 GERVAIS, Pierre, PERETZ, Pauline, STUTIN, Pierre, Le dossier secret de l'affaire Dreyfus, Paris, Alma, 2012. 
Un altro aspetto analizzato dagli stessi autori sopra citati è la presunta relazione omosessuale segreta tra Schwartzkoppen e Panizzardi, che sarebbe durata dal 1893 al 1896. Si firmavano entrambi Alexandrine e Maximilliane e ancora oggi non si sa con certezza chi abbia scritto la famosa lettera Ce canaille de D. (peraltro un falso o quanto meno post datata).

Ma ciò che diede avvio all'intera vicenda, il 27 settembre 1894, fu il ritrovamento del bordereau - anche se sarebbe meglio chiamarla "lettre-missive", visto che venne denominata in tal modo fino al 1898 - e la sua successiva consegna all'Ufficio di Statistica.

Il vero colpevole del tradimento era Esterhazy, ma Dreyfus era un ufficiale di artiglieria, stagista (cioè le supposte caratteristiche del reo), ma soprattutto ebreo: proprio per questo era ritenuto colpevole.

Nel contesto dell'epoca - nazionalista, omofoba e antisemita - gli ufficiali erano certi della colpevolezza di Dreyfus in quanto ebreo in combutta con omosessuali (Schwartzkoppen e Panizzardi) e, forse, a sua volta omosessuale. Con questa correlazione, cioè ebraismo più omosessualità, la sua colpevolezza non era dubitabile. In conclusione, secondo Gervais, Peretz e Stutin il bordereau è molto periferico rispetto alla somma delle accuse rivolte a Dreyfus; i militari volevano farlo passare per traditore, insistendo sulla omosessualità di Schwartzkoppen e Panizzardi.

Relativamente alle procedure, il processo Dreyfus del 1894 fu simile ai processi medievali per eresia: infatti, in entrambi il contenuto dell'accusa indicibile (il "nefandum") o l'insieme degli atti innominabili di cui si era accusati imponeva al processo la procedura straordinaria.

Vi era, inoltre, anche una similitudine di contenuti: così come la sodomia fu equiparata dal IX secolo all'eresia, allo stesso modo i giudici intesero accusare Dreyfus proprio di questo reato. Era dunque una struttura accusatoria degna dell'inquisizione medievale, quella che pesava sul capitano: ebreo, traditore e legato a dei sodomiti.

Accusatori e difensori nascosero la dimensione omosessuale dell'accusa per ragioni di decenza e di diplomazia (per evitare tensioni con Germania e Italia). La presunta lotta contro lo spionaggio si mutò, in definitiva, in una crociata morale e politica.

Il processo non fu acceso o violento. L'accusa utilizzò tutte le false informazioni raccolte sul conto del capitano: giocatore incallito, pessimo marito, infingardo. I rapporti della Prefettura erano in realtà quasi tutti favorevoli a Dreyfus, ma ad essi nel processo non venne fatto riferimento alcuno: la difesa, addirittura non ne era a conoscenza; sarebbero stati "ritrovati" solo nel 1903. 
In sostanza non si era in possesso che di una prova: il bordereau. Occorreva perciò rafforzare il capo di imputazione; così Henry si rimise in moto, creò (assieme a Paty) il falso dossier di cui si è detto ${ }^{19}$ e lo consegnò alla Corte.

Il 22 dicembre il processo giunse a conclusione; i giudici si ritirarono. Dopo un'ora e cinque minuti di riunione, il Presidente Maurel proclamò la sentenza: l'accusato era stato ritenuto colpevole.

Passiamo ora al processo del 1899; su di esso relazionano numerosi giornali presenti nel fondo.

Durante i trenta giorni di dibattimento sfilarono davanti ai giudici, e ad un centinaio di giornalisti venuti da tutto il mondo, ben cento testimoni militari (tra cui molti generali e ministri) e venti civili. Occorre tenere conto che comparirono davanti ad un tribunale militare, composto cioè non da civili, ma da loro sottoposti.

Vennero riproposti tutti i vecchi documenti probatori, anche se la stessa Corte di Cassazione li aveva oramai riconosciuti come falsi ed infondati: niente era cambiato, perciò, da cinque anni prima.

Ma il momento più atteso fu la deposizione di Mercier del 12 agosto; il generale parlò per quattro ore e infine così concluse:

Le général Mercier: «Eh bien non! Ma conviction, depuis 1894, n’a pas subi la plus légère atteinte. Elle s'est fortifiée par l'étude plus complète et plus approfondie de la cause. Elle s'est fortifiée aussi de l'inanité des résultats obtenus pour prouver l'innocence du condamné de 1894, malgré l'immensité des efforts accumulés, malgré l'énormité des millions follement dépensés...» ${ }^{20}$.

Alla conclusione del dibattimento sembrava certa un'assoluzione, grazie alla cosiddetta "minoranza di favore" (sarebbero risultati sufficienti tre voti favorevoli su sette); venne, invece, successivamente pronunciata una nuova condanna. Dopo le arringhe degli avvocati, Dreyfus fece un'ultima dichiarazione: «Se ho potuto sopportare cinque anni di terribili torture, fu perché pensai di dover rendere a me ed ai miei figli l'onore. Credo di aver raggiunto questo scopo grazie alla vostra lealtà ed alla vostra giustizia. Non ho altro da aggiungere! ${ }^{21}$.

Quindi il Consiglio si ritirò subito per deliberare, ma i giudici militari credettero a Mercier; il 9 settembre il presidente Jouaust lesse il verdetto: «Il Consiglio di Guerra, a

19 RIZZONI, Gianni, Cronaca illustrata del caso che ha sconvolto la Francia, Milano, Mondadori, 1994, p. 69.

${ }^{20}$ Déposition de M.le Général Mercier devant le Conseil de Guerre de Rennes, le 12 Août 1899, Paris, Imprimerie Noizette et C., 1899, pp. 127-128.

${ }^{21}$ Fondo Paulucci - Biblioteca A. Saffi di Forlì, «La Stampa», 10 settembre 1899. 
maggioranza di cinque voti contro due, dichiara: 'Sì, l'accusato è colpevole'. Gli vengono riconosciute le circostanze attenuanti. In base a ciò, il Consiglio condanna Alfred Dreyfus alla pena di dieci anni di carcere e alla degradazione ${ }^{22} »$.

Le reazioni, sia in Francia che in tutta Europa, suscitarono uno sdegno generale, anche se non mancarono gli antidreyfusardi irriducibili («La Libre Parole», ad esempio, titolò Le Traître condanné. Vive l’Armée! Vive la France! A bas les juifs!'23).

Già l'11 settembre Mathieu Dreyfus avanzò l'idea della grazia, ma ciò avrebbe significato accettare, di fatto, il giudizio emesso a Rennes. A favore della proposta si schierarono Lazare e Reinach, contrari furono invece Clemenceau, Labori e Jaurès; infine, però, anch'essi si convinsero.

Si giunse così alla concessione della grazia, il 19 settembre, che riconobbe a Dreyfus, non la cancellazione della pena, ma un suo "condono".

La notizia, come è ovvio, non venne accolta positivamente dagli antidreyfusardi: «Le Soir» titolò a tutta pagina Le traître Dreyfus gracié. Outrage à l'Armée ${ }^{24}$.

L'ultimo atto dell'intera vicenda fu - come già anticipato - la riabilitazione del 12 luglio 1906. Ormai Dreyfus non era più il simbolo di una battaglia politica per la giustizia contro le forze oscurantiste che insidiavano la Repubblica, ma un uomo in carne ed ossa. Il caso Dreyfus diviene, cioè, il “caso di Dreyfus”.

Nella causa di revisione Dreyfus fu assistito dall'avvocato Mornard; le Camere Riunite deliberarono infine l'innocenza del capitano all'unanimità; a maggioranza, invece, venne cassato il giudizio di Rennes "senza rinvio", cioè senza che l'ufficiale dovesse di nuovo comparire in giudizio. La sentenza venne letta il 12 luglio 1906 da Ballot-Beaupré, presidente delle Camere Riunite, il quale dichiarò, relativamente alla condanna di Rennes, «che è per errore e a torto che tale condanna è stata pronunciata» 25 .

Il 21 luglio 1906 si tenne, infine, la cerimonia ufficiale di riabilitazione di Dreyfus.

\section{Il ruolo degli avvocati nell'affaire}

La figura dell'avvocato che usa il diritto come arma contro il potere nasce nell'Illuminismo, con il già citato affaire Calas. Va notato, però, che nel caso Dreyfus vi fu uno scarso coinvolgimento degli avvocati, a differenza di quanto avvenne per gli

22 RIZZONI, Gianni, Cronaca illustrata del caso che ha sconvolto la Francia, cit., p. 210.

${ }^{23}$ Fondo Paulucci - Biblioteca A. Saffi di Forlì, «La Libre Parole», 9 settembre 1899.

24 Fondo Paulucci - Biblioteca A. Saffi di Forlì, «Le Soir», 20 settembre 1899.

25 SILVESTRI, Agnese, Il caso Dreyfus e la nascita dellintellettuale moderno, Milano, Franco Angeli, 2012. 
intellettuali; in sintesi, si può infatti affermare che l'affaire Dreyfus rappresentò il punto più alto dell'alleanza tra scena giudiziaria (con il processo che diventò occasione di disvelamento e di apertura allo spazio pubblico) e dibattito intellettuale, nell'ambito del cosiddetto modello liberale ${ }^{26}$.

Il primo processo contro Dreyfus si svolse a porte chiuse. Malgrado la condanna subita dal suo assistito, si segnalarono le grandi capacità dell'avvocato difensore, vale a dire Charles Demange. Il suo impegno non fu certo scevro da rischi, anzi: per aver difeso il "traditore" Dreyfus, egli venne infatti emarginato persino dall'Ordine degli avvocati. Ancor peggio andò a Fernand Labori, l'altro avvocato che difese Dreyfus nel corso del secondo processo istruito contro quest'ultimo, vale a dire quello celebrato a Rennes nel 1899. In tale occasione, Labori fu addirittura vittima di un attentato.

Com'è facilmente intuibile, l'attività di questi avvocati appare svolta in difesa dei diritti dell'individuo, a differenza di quanto accade per i magistrati (sia civili che militari) che sostennero i vari processi in cui si articolò l'affaire, i quali si adoperarono a favore del regime in nome di quella che Paulucci chiama "la ragion di stato".

Secondo Christophe Charle ${ }^{27}$, il sostegno tributato da Demange, Labori, Clemenceau e Leblois (consigliere e confidente di Picquart) all'“innocente" costituiva una rottura sociale e una scommessa quasi insensata, vista la forza di pressione politica che si esercitava su di essi. La loro non era infatti una scelta razionale, ma etica, in accordo ai rispettivi valori; Dreyfus era moralmente innocente, quindi non poteva che esserlo anche realmente. Era, questo, un ragionamento inverso rispetto a quello degli antidreyfusardi: costoro infatti ritenevano Dreyfus colpevole eticamente e realmente e per dimostrare ciò ritenevano di poter fare ricorso anche a false prove.

In sintesi, conclude Charle, non è eccessivo affermare che l'affaire Dreyfus segnò senza dubbio la fine dell'egemonia degli avvocati nella vita pubblica francese, a vantaggio degli intellettuali.

Tra i numerosi avvocati antidreyfusardi si segnalano infine Tézanas du Montcel (che difese Esterhazy) e Joseph Menard (avvocato del du Paty).

\footnotetext{
${ }^{26}$ ISRAËL, Liora, Le armi del diritto, cit., pp. 40, 41, 49.

${ }_{27}$ CHARLE, Christophe, Le déclin de la République des avocats, in BIRNBAUM, Pierre (dir), La France de l'affaire Dreyfus, Paris, Gallimard, 1994, pp. 56-86.
} 


\section{Il fondo Paulucci e il diario del 1898}

Giovanni Tassani, curatore del diario e del Fondo Paulucci, ha sottolineato come «la pagine di un diario a volte possono essere la chiave che consente di penetrare, a chi legge a distanza di tempo, un'epoca e un personaggio» ${ }^{28}$.

Nel 1898 Paulucci era un giovane trentasettenne segretario di ambasciata; egli riferiva dunque dell'affaire Dreyfus in presa diretta, fornendoci così informazioni non solo sull'opinione pubblica, ma anche sulla situazione politica ad essa correlata.

\subsection{Il diario del 1898}

Fin dal 3 gennaio 1898 Paulucci si occupò, nelle pagine del suo diario, dell'affaire.

Il 16 gennaio egli annotò di essere venuto a conoscenza del fatto che Esterhazy (per pagare dei debiti) addirittura già dall'agosto 1894 percepiva compensi dall'addetto militare tedesco Schwartzkoppen in cambio di importanti documenti segreti.

Ma la vicenda si complicò ulteriormente perché anche Zola venne citato, in seguito alla pubblicazione dello $J$ 'accuse, per diffamazione. Egli non ebbe però la possibilità di dimostrare quanto aveva affermato nei suoi scritti e il 23 febbraio giunse la condanna (per Paulucci scontata, a quanto si legge nel diario): un anno di prigione e tremila franchi di multa.

Nel frattempo, ci informa ancora Paulucci, Panizzardi era stato costretto a lasciare Parigi per trasferirsi a Berna e, da lì, a Roma. Contemporaneamente si verificò il tentativo di ottenere la revisione del processo Dreyfus; una prima istanza, in tal senso, fu però osteggiata dal ministro della Guerra, Cavaignac: per il capitano francese la libertà era ancora lontana, notava amaramente Paulucci ${ }^{29}$.

Il 13 luglio l'Affaire ritornava d'attualità per via dell'arresto dell'Esterhazy, a carico del quale vi erano numerose prove. Purtroppo, aggiunge Paulucci, assieme a costui si trovava in carcere anche l'innocente Picquart (definito "un eroe").

Sempre in luglio, il 18, venne annotata la nuova condanna inflitta a Zola, il quale fu costretto a fuggire in Inghilterra per evitare l'arresto. In agosto Paulucci registrò invece altre battute d'arresto per i dreyfusardi: il tenente colonnello Paty de Clam (obiettivo principale dello $J$ 'accuse) venne prosciolto dall'accusa di falso, mentre il 13, Esterhazy

28 PAULUCCI DI CALBOLI, Raniero, Parigi 1898 con Zola, per Dreyfus. Diario di un diplomatico, cit., p. 3 .

29 Ibidem, p. 162. 
fu addirittura liberato. Solo a fine mese, il 31, Paulucci potè riportare una buona notizia: il colonnello Henry - che si sarebbe suicidato il $1^{\circ}$ settembre - venne arrestato per aver fabbricato le false prove che portarono alla condanna di Dreyfus.

Ma l'euforia dei dreyfusardi durò pochissimo: il 7 settembre, infatti, il Ministro della Giustizia, Sarrien, ribadì come non vi potesse essere il minimo dubbio sulla colpevolezza di Dreyfus; malgrado tutto questo, il 26 settembre Paulucci appuntò che il Consiglio dei Ministri aveva deciso di operare una revisione del processo Dreyfus.

Un tentativo di bloccare il provvedimento da parte del governo Brisson (che cadde il 27 ottobre) fu scongiurato, così il 28 ottobre la Cassazione riprese in mano le sorti di Dreyfus, per poi annunciare definitivamente l'accoglimento della revisione il 30 ottobre.

Il governo, nota ancora Paulucci, a questo punto si trovava tra l'incudine della giustizia ed il martello dell'esercito; Dupuy e Freycinet «non sanno a che santo votarsi per poter ragionevolmente risolvere l'affaire e l'incidente Picquart» ${ }^{\circ}$. In soccorso di quest'ultimo la Cassazione, il 9 dicembre, riconobbe la connessione con l'Affaire: era un trionfo per i dreyfusardi. Il diario si chiude perciò, il 30 dicembre, con la certezza da parte di Paulucci che la revisione non avrebbe potuto più essere messa in dubbio ${ }^{31}$.

\subsection{Le opinioni di un diplomatico}

Il diplomatico forlivese non esitò, nel suo diario, a prendere posizione con una serie articolata di interventi significativi.

A partire dal 10 gennaio, Paulucci definì il processo Dreyfus un'infamia, per poi affermare con decisione: «Non avrei mai pensato a tanta bassezza umana! Tre periti giurano che [quella sul borderau] non è la scrittura di Esterhazy, che ne uscirà agnus sine macula» ${ }^{32}$.

Il 15 gennaio il diplomatico incontrò per la prima volta Zola, presso la redazione della «Revue»; era stato il direttore della stessa, Finot, a presentare i due: il forlivese definì l'illustre interlocutore «uomo onesto, intelligente, integro, un vero carattere!» ${ }^{33}$. Da questo momento rimase sempre al suo fianco.

Due giorni dopo, il 17 gennaio, Paulucci scrisse: «Se davvero fossero così le cose maledirei la giustizia», riferendosi alla possibilità che un’ipotetica deposizione di

\footnotetext{
30 Ibidem, p. 260.

${ }^{31}$ Ibidem, p. 275.

32 Ibidem, p. 39.

33 Ibidem, p. 43.
} 
Schwartzkoppen non sarebbe accettata dal tribunale come prova valida a discolpa di Dreyfus.

Paulucci, poi, non lesinava giudizi sprezzanti verso coloro che non reputa degni di stima. Era il caso di Panizzardi, bollato con un lapidario: «Preferisce evidentemente i propri comodi e la propria tranquillità a qualsiasi menomo sacrificio» ${ }^{34}$. Pochi giorni dopo, il 4 febbraio, quando Zola invitava vanamente l'addetto militare a parlare, aggiunse: «Panizzardi non ha più alcun rimorso di coscienza, se pure ne ha mai avuto. Il desiderio di restare qui tranquillo e contento gli vela il triste spettacolo del deportato innocente ${ }^{35}$. In seguito, il 16 aprile, scriveva: «Panizzardi è partito jersera per Berna... Purché anche laggiù non faccia delle sciocchezze» ${ }^{36}$.

Le critiche non venivano risparmiate nemmeno alle istituzioni: il 14 febbraio Paulucci annotava, per esempio, «la niuna base solida del governo repubblicano e la poca serietà dei dirigenti l'esercito» 37 .

Del resto, la consapevolezza del suo coinvolgimento totale era ben presente in lui, tanto che il 15 febbraio ammetteva, a proposito del processo contro Zola: «Io mi interesso tanto a questo processo che non sono buono a fare altro e lascio da parte qualsiasi lavoro!». Per questo motivo è comprensibile il suo sfogo all'indomani del verdetto di colpevolezza di Zola, emanato il 23 febbraio: «[...] infame verdetto pronunziato stasera contro lo Zola. Un anno di prigione e tremila franchi di multa» ${ }^{8}$. Tutto ciò veniva accompagnato da «una pessima ed immonda stampa, che è riuscita ad avvelenare la coscienza pubblica»39, come notava argutamente Paulucci. Ai suoi occhi, si legge alla data 24 febbraio, «tutto è volgare e delittuosa finzione!»40.

Ad aggravare la situazione contribuiva, inoltre, il già più volte menzionato problema dell'antisemitismo, considerato fondamentale nell'attacco a Dreyfus. Paulucci accennava ripetutamente all'argomento: il 28 febbraio scrisse di essere d'accordo con Finot, per il quale vi era primato di comprensione e generosità su ogni pregiudizio di razza, per arrivare, il $1^{\circ}$ settembre, ad affermare: «L'attitudine però degli antisemiti e dei nazionalisti fa schifo» ${ }^{11}$.

Anche nel corso del mese di marzo Paulucci trovò modo di esprimere il proprio dissenso nei confronti delle istituzioni francesi; il giorno 3 si legge: «Sembra di

\footnotetext{
34 Ibidem, p. 56.

35 Ibidem, p. 59.

${ }^{36}$ Ibidem, p. 112.

37 Ibidem, p. 66.

${ }^{38}$ Ibidem, p. 72.

39 Ibidem.

40 Ibidem, p. 73.

${ }^{41}$ Ibidem, 207.
} 
sognare!»42 perché, invece di mettere a tacere il prepotente, in Francia il potere civile non si palesa e «per poco non si eleva un monumento all’insultatore!»43.

Nel diario si alternavano vari giudizi, ora trancianti - ad esempio, quando il 5 marzo Picquart ferì Henry a duello, Paulucci non esitò ad affermare: «Ciò m’ha fatto immenso piacere. È stato il giudizio di Dio!»44 - ora quasi rassegnati e malinconici - il 4 aprile scriveva, «Ma purtroppo la verità non farà alcuna impressione, tanto più che le si farà d’intorno la cospirazione del silenzio!»45 - ora viceversa euforici - il 13 luglio si legge: «Si era detto che l'affare Dreyfus era morto e sotterrato... Altro che!»46 -, allorquando Esterhazy venne arrestato.

Un duro colpo fu invece costituito dalla nuova condanna di Zola («Che vergogna per la magistratura francese!»47 riportava il 18 luglio); Paulucci si lasciò poi andare, il 6 agosto, ad amare considerazioni, quali: «E la coscienza pubblica così offesa non insorge! C'è da vergognarsi d'esser uomo! Ci riconcilia coll'umanità il sentire qualche grido eroico di protesta in mezzo a tanta viltà [...]»48; oppure, il 13 agosto troviamo la notizia della liberazione di Esterhazy: «Viva la Francia, abbasso la magistratura francese!»49, mentre il 20 agosto il diplomatico forlivese non potè fare a meno di scrivere: «Ma ci vuol altro che la verità quando si tratta di un popolo cieco, sordo e balordo!»50.

Anche nelle occasioni in cui gli eventi parevano evolvere in senso positivo (come il 31 agosto: «Henry arrestato»51) Paulucci rimase legato ad una visione realistica e lungimirante («L'Henry sarà considerato come un martire, un povero individuo che ha sacrificato il proprio onore per far finire una buona volta questo maledetto affare che turbava la pace dell'esercito e del paese ${ }^{52}$.) in cui trovavano spazio il rammarico (13 settembre: «Ma intanto il povero Picquart fa il suo sessantatreesimo [sic!] giorno di prigione. Che infamia!»53) e la soddisfazione (18 settembre: «Con che piacere ho visto [...] che la revisione è decisa»54; oppure, il 26 novembre: «Finalmente lo spirito pubblico, che parea assopito, si risveglia ed oggi abbiamo una vera levata di scudi,

\footnotetext{
42 Ibidem, p. 78.

43 Ibidem.

44 Ibidem, p. 80.

45 Ibidem, p. 103.

46 Ibidem, p. 173 .

47 Ibidem, p. 177.

48 Ibidem, p. 189.

49 Ibidem, p. 194.

5o Ibidem, p. 198.

${ }^{1}$ Ibidem, p. 204.

$5^{2}$ Ibidem, p. 205.

53 Ibidem, p. 214.

54 Ibidem, p. 216.
} 
contro il responso dell'autorità militare nella fissazione della data del processo Picquart»55).

Ma è la speranza che, il 30 dicembre, chiude significativamente il diario, malgrado l'ennesimo contrattempo registratosi: «[...] non credo che la revisione possa essere messa in dubbio, sarà soltanto un altro bastone nelle ruote che ritarderà la famosa marcia della verità ${ }^{56}{ }^{\prime}$.

\subsection{Il ruolo della stampa nell'affaire}

Nel diario di Paulucci si ritrova anche una puntuale riflessione sul ruolo giocato dalla stampa nell'Affaire. Gli schieramenti risultano abbastanza netti, anche se qualche giornale passò dalla posizione dreyfusarda a quella antirevisionista: è il caso del «Le Figaro» che, dopo aver ospitato sulle proprie colonne articoli di Zola, l'11 gennaio si schierò dalla parte di Esterhazy. Per questo il forlivese il 20 gennaio lo definì «quel giornalaccio»57 (al pari del «Le Croix», il 3 settembre) salvo poi annotarne il ritorno convinto su posizioni favorevoli all'innocenza di Dreyfus (si veda la pagina del 18 febbraio).

Altrettanto importante risultava il giudizio dei lettori, così come traspare da quanto annotato il 7 marzo, in riferimento ad una lettera indirizzata da uno di essi al Finot, per contestare l'attitudine contraria al sentimento nazionale della «Revue».

Il «Jour» viene definito il 10 aprile «immondo giornale» 58 , con un giudizio simile a quel «sozza stampa» tributatogli poi il 14 aprile; «Le Soir» e «Le Gaulois», il 2 luglio, sono etichettati entrambi come giornali antirevisionisti in nome dell'ordine.

L'8 luglio Paulucci fa riferimento a una «ridda festosa delle canaglie della penna»59, cui fa da contraltare la celebrazione di un articolo «della brava Severine»60, il 22 luglio, a proposito del processo Zola, e di una bella serie di interventi da parte di Jaurès (scrive l'11 agosto: «L'articolo del Jaurès [...] è stupendo. Lo trovo d'assai superiore al primo [...]. Che peccato che «La Petite Republique» non abbia la tiratura del «Petit Journal»! [solo 45 mila copie contro il milione del secondo, ndr]. Con articoli così convincenti, che parlano al cuore e alla mente, l'opinione pubblica cambierebbe in pochi giorni! $\left.{ }^{61}{ }^{\prime}\right)$

\footnotetext{
55 Ibidem, p. 255.

${ }^{5}$ Ibidem, p. 275.

57 Ibidem, p. 46.

${ }^{8}$ Ibidem, p. 108.

59 Ibidem, p. 168.

60 Ibidem, p. 180.

${ }^{61}$ Ibidem, p. 193.
} 
anche se un po' troppo lunghi («Quale operaio mai può consacrare un pajo d'ore a quelle letture?»62 si chiede il 19 agosto).

Anche in questo caso, il finale del diario è a tinte rosee: il 10 novembre Paulucci registrò con piacere: "Adesso i camelots, invece delle caricature di Zola, vendono l'“Histoire d'un innocent" col trionfo finale del Dreyfus»63.

\subsection{Il peso dell'opinione pubblica}

L'opinione pubblica apparve fortemente condizionata sia dall'attività governativa, sia dalla propaganda della stampa ad essa legata. Paulucci riferisce, per esempio, in data 17 febbraio, di una possibile insurrezione della plebe parigina (ovviamente istigata) in caso di assoluzione dello Zola, finalizzata a «sgozzare tutti gli Ebrei!» 64.

L’opinione pubblica generò anche atteggiamenti punitivi: così, il 15 marzo Paulucci riporta come le vendite dell'ultimo romanzo di una trilogia di Zola (il Paris) risultaono in netto calo rispetto ai dati ottenuti dai due precedenti lavori (solo 1.000 copie contro 8.00o), a causa della campagna denigratoria orchestrata contro di lui.

La speranza che, malgrado continuasse la campagna anti Dreyfus - «la corrente non la si arresta più»65 (2 settembre) - venne mitigata il 5 e poi il 7 settembre dall'atteggiamento di chiusura sia del governo che dell'esercito; il ministro della Giustizia Sarrien affermò infatti che non vi era il minimo dubbio sulla colpevolezza di Dreyfus. «Ma son proprio tutti ciechi?» si chiedeva Paulucci.

Il 23 e il 24 novembre nel diario c'è spazio anche per il compatimento del Picquart, allora osteggiato dal governo. Ciò era comunque troppo anche per il Senato, che insorse a favore di Picquart contro l'autorità militare: «Finalmente lo spirito pubblico, che pareva assopito, si risveglia» ${ }^{66}$, annunciò trionfante Paulucci il 26 novembre. Non poteva esservi comunque spazio per i trionfalismi perché, come aggiungeva poche righe più sotto: «Sfortunatamente la maggioranza della stampa è sempre antirevisionista e più esaltata che mai!». La convinzione, però, era che «la rivoluzione morale non potrà certo arrestarsi. Governo ed esercito, se vi si opporranno, saranno abbattuti. Non ho adesso il minimo dubbio sul successo finale e sul completo trionfo della verità» ${ }^{67}$ E ancora: «Grazie a Dio, il cambiamento nell'opinione pubblica si fa..., la macchia famosa

\footnotetext{
62 Ibidem, p. 198.

63 Ibidem, p. 196.

64 Ibidem, p. 67.

65 Ibidem, p. 206.

66 Ibidem, p. 255.

67 Ibidem, p. 256.
} 
d'olio si allarga sempre sempre di più!» ${ }^{68}$. Infatti, il 2 dicembre Paulucci annotava: «Intanto il plebiscito per Picquart continua... Il buon colonnello può dormire tra due guanciali» ${ }^{69}$, mentre il 9 dicembre scrisse: «Trionfo dei Dreyfusards»70.

Finalmente cominciavano ad intravedersi la giustizia e la verità.

\subsection{Il Fondo Paulucci}

Come afferma Marco Grispigni ${ }^{71}$, il fondo evidenzia la perspicacia di Paulucci, che colse subito nell'Affaire non un semplice caso di spionaggio, ma un'intricata vicenda politica, capace di incidere in maniera periodizzante sulla storia nazionale francese: con esso l'Ottocento veniva messo alle spalle e si è chiudeva la porta su un secolo ormai passato.

Il fondo è attualmente composto da 248 volumi, per un totale di 357 titoli, oltre a giornali, riviste, volantini, manifesti, cartoncini di propaganda, documenti vari, foto (39), lettere e appunti sparsi (4 cartelle). Il Fondo librario contiene invece 350 titoli.

Il materiale copre un arco cronologico che va dal 1894 al 1908, ma la maggior parte di esso riguarda il biennio 1898-1899. Una certa produzione antidreyfusarda utilizzò l'antisemitismo e i più classici luoghi comuni: Dreyfus venne raffigurato con un naso spropositato, oppure come una scimmia o un serpente, a seconda dei casi; Zola fu spesso rappresentato come un porco perché i suoi romanzi erano ritenuti immorali, mentre Picquart era accusato di essere omosessuale (veniva infatti chiamato "Georgette").

Di contro, in campo dreyfusardo, troviamo l'attacco alla giustizia militare e al clericalismo, sia nei giornali che nelle riviste. In gioco vi erano i principi ispiratori della Repubblica: lo scontro si poneva tra la difesa dell'ordine e la difesa dei valori etici individuali. I dreyfusardi indicavano nello Stato maggiore dell'esercito e nei gesuiti i principali nemici, che sull'onda dell'affaire Dreyfus tentavano appunto di mettere in discussione i principi stessi della Repubblica.

\footnotetext{
68 Ibidem, p. 257.

69 Ibidem, p. 260.

70 Ibidem, p. 263.

${ }^{71}$ GRISPIGNI, Marco, Origine e composizione del fondo, in GRISPIGNI, Marco (a cura di), Il fondo Paulucci di Calboli sull'affaire Dreyfus, Inventario, Bologna, Pàtron, 1997.
} 


\section{La vita, la personalità e la passione civile di Raniero De Calboli}

In conclusione, appare opportuno riportare alcune note biografiche su Raniero Paulucci di Calboli: nacque a Roma il 15 marzo 1861 dal marchese Annibale e da Mary Ann Simpkinson. Orfano dall'infanzia, viene inizialmente accudito dallo zio Amilcare, canonico della Cattedrale di Forlì. In seguito studiò giurisprudenza alla Sapienza di Roma, poi passò a Bologna, dove si laureò. Iniziò la carriera diplomatica nel 1885, come addetto d'ambasciata a Londra; poi venne trasferito a Vienna, quindi di nuovo a Londra: qui incontrò l'ambasciatore Giuseppe Tornielli Brusati, suo vero maestro (di cui sposerà, tra l'altro, la nipote Virginia). Assieme a questi, nel 1895 giunse a Parigi. Qui tra il personale diplomatico italiano figurava il colonnello Alessandro Panizzardi, addetto militare, definito dal Paulucci eccentrico e ondeggiante ${ }^{72}$. Il rapporto con quest'ultimo, malgrado i caratteri opposti, sarebbe divenuto più disteso in particolare dopo l'Affaire.

In seguito, aderì alla rivista «La Revue Des Revues», con cui iniziò a collaborare stabilmente. Tale adesione avrebbe dato al diplomatico forlivese informazioni in più rispetto a quelle d'ambasciata e gli avrebbe fatto toccare con mano il ruolo dell'informazione e dell'opinione pubblica. Infatti, nel 1897, denunciò la tratta dei piccoli italiani per lavori logoranti, con magro salario. Si avvicinò anche al mondo intellettuale ebraico, spogliandosi di vecchi pregiudizientrando così in contatto con Zola.

Nel 1896 l'affaire era già esploso; in esso venne coinvolto anche Panizzardi, che confidò però a Paulucci la propria estraneità ai fatti; la moglie e il fratello di Dreyfus erano certi dell'innocenza del congiunto: il processo doveva essere riaperto.

Paulucci registrò tutti gli avvenimenti relativi all'affaire nel suo diario del 1898; successivamente, nel 1930, avrebbe raccontato che la passione per il caso Dreyfus era nata nel 1897: da allora aveva raccolto numerosissimi documenti, rilegandoli in verde (il colore della speranza, poiché si voleva salvare un innocente) che poi sarebbero andati a comporre il fondo.

Dopo Parigi, Paulucci si sarebbe recato in Portogallo, Svizzera, Giappone e, infine, in Spagna; morì nel 1931.

72 PAULUCCI DI CALBOLI, Raniero, Parigi 1898 con Zola, per Dreyfus. Diario di un diplomatico, cit., p. 11. 


\section{* L'autore}

Francesco Gioiello è nato a Forlimpopoli (FC) nel 1990. Laureato con lode in Lettere moderne, frequenta il primo anno di Scienze storiche a Bologna. Ha vinto nel 2013 il concorso bandito in occasione del 25 aprile dal Comune di Forlì, con un saggio sul ruolo delle donne nella Resistenza.

URL: < http://www.studistorici.com/progett/autori/\#Gioiello >

\section{Per citare questo articolo:}

GIOIELLO, Francesco, «Raniero Paulucci di Calboli e l'affaire Dreyfus», Diacronie. Studi di Storia Contemporanea : Processo penale, politica, opinione pubblica (secoli XVIII-XX), 29/08/2013,

URL:< http://www.studistorici.com/2013/08/29/gioiello_numero_14/ >

Diacronie Studi di Storia Contemporanea $\mathcal{3}$ ww.diacronie.it

Risorsa digitale indipendente a carattere storiografico. Uscita trimestrale. redazione.diacronie@hotmail.it

Comitato di redazione: Marco Abram - Jacopo Bassi - Luca Bufarale - Alessandro Cattunar - Elisa Grandi - Deborah Paci - Fausto Pietrancosta - Matteo Tomasoni - Luca Zuccolo 